\title{
PHYSICS IN DAILY LIFE: WINE TEMPERATURE
}

\section{L.J.F. (Jo) Hermans,}

Leiden University• The Netherlands.Email: Hermans@Physics.LeidenUniv.nl

t happens to all of us, once in a while: We go to open up a bottle of wine only to discover that the temperature isn't right. If it is a red wine and it's too cold, it's easy: we can simply put it in the microwave oven for a few seconds (don't tell the wine maker). But if it's a white wine that is much too warm, we have a problem. All we can do is put the bottle in the refrigeratorand be patient. How long will it take for the wine to reach the desired temperature?

Being physicists, we realize that the answer is determined by exponential decay of the temperature difference between bottle and refrigerator, with the time constant being the thermal relaxation time of a bottle of wine in air. This is not exactly a problem treated in the textbook, but it is easily solved. The thermal relaxation time simply equals $R C$, the product of thermal resistance $R$ and heat capacity $C$. Since $C$ can be well approximated by the value of water, all we need to find out is the thermal resistance $R$ of the glass. This may sound cumbersome for an exotic shape like a wine bottle, but an approximation in terms of a parallel-plate geometry will do. Therefore we put $R$ $=d / k A$, where $d$ is the glass thickness, $k$

its thermal conductivity coefficient and $A$ its total outer surface area.

The calculation is easy to do, with $d$ just over $3 \mathrm{~mm}$ as determined from the weight of an empty bottle, its outer surface area and the density of glass. The resulting relaxation time is found to be almost exactly 3 minutes.
Three minutes! Or even less if we include radiation! That can't be right, as we know from experience. And indeed, it isn't. The reason is that we have grossly underestimated the value of $R$. We must include the thin layer of air surrounding the bottle, which is effectively convection-free, and in which thermal transport relies on conduction. This layer represents a much larger thermal resistance than the glass does. The 3 minutes just calculated must be considered a lower limit. The real value of the relaxation time of a wine bottle in the refrigerator is found to be about 3 hours, corresponding to an effective air layer of a few $\mathrm{mm}$ thick.

Of course we could speed up the cooling process by putting the bottle in iced water rather than air. If natural convection in

water and wine is sufficiently effective, we may approach the limit calculated above.

But there is a better way to cool our wine: use a commercial 'cooling jacket' which contains a cooling gel that has a large latent heat capacity. The advantage is that it may be pre-cooled to far below $0^{\circ} \mathrm{C}$ and provides a good thermal contact with the wine bottle.

Obviously, this trick works for any bottle, regardless of its contents. But in view of the heavy brain work we have just done, it seems only fair to treat ourselves to a delicious Pouilly-Fumé from the Loire, or a Pinot blanc from Alsace. At just the right temperature... after only 8 minutes.

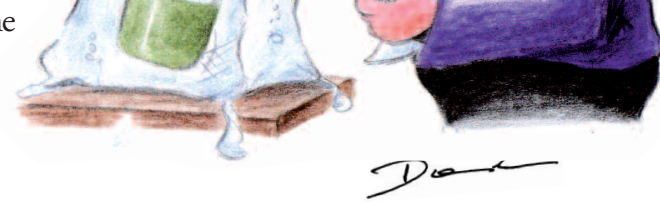

